\title{
PROBLEMAS Y METODOS PARA LA ENSEÑANZA DEL INGLES COMO SEGUNDA LENGUA A LOS HABLANTES DEL MEK-A-TEL-YU EN LA PROVINCIA DE LIMON
}

Fernando Wright $M$.

\section{El habla criolla limonense o mek-a-tél-yu}

En este estudio presentaré un esbozo descriptivo del inglés criollo conocido como mek-a-tél-yu (M) que se habla en la provincia de Limón. Mencionaré algunos de los obstáculos que el hablante de esta lengua encuentra en el aprendizaje del inglés normativo que se enseña a nivel de secundaria en Costa Rica y sugeriré métodos y técnicas que el educador podría implementar para mejorar la enseñanza del inglés.

El mek-a-tél-yu es una lengua que toma la mayor parte de su léxico del inglés pero ni su estructura gramatical ni su fonología son las mismas de un inglés estándar. Mek-a-tél-yu, del inglés Let me tell you, significa 'Déjame decirte'.

Orígenes del mek-a-tél-yu

El mek-a-tél-yu es muy parecido al criollo jamaiquino por causa de que la mayoría de los inmigrantes que llegaron de las Antillas a Limón a partir de 1872 para trabajar en la construcción del ferrocarril y la explotación bananera, eran jamaiquinos. Según datos muy recientes, el mek-a-tél-yu es hablado por casi todos los descendientes de estos antillanos que son cerca de 40.000 personas (Herzfeld, 1978).

Por lo menos son tres las lenguas que existen en Limón, actualmente: el español tico -que es el idioma de prestigio-, el inglés estándar limonense (SL) - que se considera la forma culta, y que coincide con la variante que sirve de modelo en Jamaica-, y el mek-a-tél-yu (M) - que se considera como habla no cultivada.

\section{Problemas para aprender el inglés}

Los hablantes del $\mathrm{M}$ no tropiezan con ningún obstáculo para hacerse bilingües en el español. Desde la infancia los niños despliegan una habilidad extraordinaria para expresarse en los dos idiomas. Pero el inglés que se enseña en los colegios, una variedad del inglés norteamericano, y que se considera como una forma cultivada, "buena", plantea un problema más serio. Como el hablante del criollo reconoce y entiende un alto porcentaje del inglés estándar, el refuerzo del aprendizaje que se deriva de la satisfacción íntima de dominar un nuevo elemento, en este caso una lengua nueva, se reduce considerablemente.

El hablante del criollo practica los patrones estructurales del inglés en clase, pero invariablemente retorna al $\mathrm{M}$ fuera de las aulas. El educador inconsciente de los problemas lingüísticos de sus alumnos puede cometer arbitrariedades. Debemos evitar que sucedan incidentes como el que ocurrió hace algunos años en una de las escuelas que hay para indígenas en la provincia de Puntarenas. En vista de la dificultad de encontrar maestros indígenas, es prácticamente inevitable que los maestros de dichas escuelas sean de la Meseta Central y por lo tanto hablantes de español. Cuenta el Dr. Jack Wilson (1974) que en una oportunidad le contó un informante indígena que durante toda su vida había sentido vergüenza de ser bribri porque cuando entró a primer grado en la escuela indígena, el maestro les había dicho a los alumnos que su principal meta consistía en obligar a los bribris a que olvidaran su "dialecto bárbaro" como primer paso hacia su conversión a seres civilizados. En Limón, en los años 50, se pretendió eliminar las "escuelitas inglesas" y se trató de prohibir al costarricense de ascendencia afrocaribeña hablar su lengua materna.

El mek-a-tél-yu se preserva en Limón para intercambios personales entre sus hablantes $y$ parece inevitable su eventual extinción a causa del estigma social e intelectual asociado a él. Por lo general una lengua criolla no es una lengua prestigiosa. El $\mathrm{M}$ es considerado como inglés "malo" y el inglés estándar es inglés "bueno".

$\mathrm{La}$ existencia de una lengua criolla en contacto con la lengua que ha servido como fuente principal de su material léxico lleva, por lo general, al establecimiento de lo que se ha dado en llamar un continuum o continuo entre aquella y ésta. Herzfeld dice que "el habla criolla limonense se 
puede describir como un continuo criollo-estándar con el criollo en un extremo y el inglés estándar limonense en el extremo opuesto, coexistiendo ambos en medio de una población hispano-hablante". (Extracto traducido).

Un ejemplo de este continuo se aprecia seguidamente:

1. a im kil di suorá

2. iz im kil di suorá

3. izí kil di suorá

4. it woz im kil di suorá

5. it woz i dat kil di suorá

6. it woz hi ðat kild ðəsuorá

Muy pocas personas usarían todas estas variantes. Normalmente hacen uso de dos o tres de ellas. Abstrayéndonos de los aspectos fonológicos, la única diferencia entre la última oración y el inglés modelo jamaiquino o norteamericano está en el vocabulario: suorá es término típicamente limonense para designar a la zorra. Desde el punto de vista fonológico, en las oraciones (5) y (6) el sonido vocálico en đat es también típico, pero el inglés que se enseña demanda/ae/, sonido inexistente en el inglés limonense.

Esta gradación de variantes que existe entre el criollo y el estándar dificulta la enseñanza del inglés estándar. Esta situación se puede comparar con el hispano-hablante que a menudo comete menos errores al hablar inglés que al hablar portugués, porque el español es mucho más parecido al portugués que al inglés.

Casi todos los limonenses de ascendencia afrocaribeña hablan M-SL. Sin embargo, la presencia de préstamos del español en el idioma es bastante notable, especialmente entre la gente joven.

\section{EI mek-a-tél-yu como basilecto}

Para tipificar las diversas variantes que intervienen en los continua de las lenguas criollas y las respectivas lenguas estándares, se ha propuesto (Bailey, 1974 - Bickerton, 1975) la siguiente terminología: si la lengua estándar -fuente del vocabulario del criollo- se conserva como lengua dominante, podemos denominarla 'matrilecto'. El criollo entonces permanece en situación de 'acolutecto' (es decir, un satélite lingüístico del matrilecto). El criollo normalmente se nutre del matrilecto, constituyéndose en una gradación de sistemas sobrepuestos en el cual el más alejado del matrilecto se llama 'basilecto'. El basilecto se recriolliza con el matrilecto produciendo un sistema más próximo al matrilecto. Este proceso puede repetirse constantemente de manera que cada sistema más cercano al matrilecto sigue recriollizándose con el matrilecto, creando un nuevo sistema todavía más cercano a éste. Cuando esta cadena de sistemas sobrepuestos entre el basilecto original y el matrilecto se completa, el sistema más cercano al matrilecto se denomina 'acrolecto', y los sistemas entre el acrolecto y el basilecto en la gradación son llamados 'mesolectos'.

Si aplicamos esta terminología al caso que nos ocupa, las variantes del criollo limonense más alejados en el continuum del inglés estándar limonense, es decir las variantes basilectales, integran el fenómeno para el cual en la terminología popular se emplea por antonomasia el término 'mek-a-tél-yu'.

Las variantes mesolectales y acrolectales son la necesaria transición por la cual los hablantes pasan, al ir asimilando las formas del inglés estándar limonense, teniendo como punta de partida las variedades basilectales. Se da en este proceso un reemplazo cada vez mayor de las formas basilectales en favor de las estándares.

El habla criolla limonense que se describe en este estudio es lo que De Camp denomina "un tipo abstracto ideal", o sea un compuesto de todas las características basilectales del idioma. Muy pocos limonenses practican de manera absoluta esta combinación, que en el continuum constituye el polo opuesto al inglés estándar. Adopto esta posición por cuanto estimo que el educador debe conocer y tratar situaciones extremas, y pareciera que los mejores resultados se alcanzan sólo cuando todas las posibles divergencias de la lengua se toman en cuenta.

\section{Fonemas del mek-a-tél-yu}

A nivel fonológico existen 19 consonantes, 8 vocales y 4 diptongos. Este estudio presenta dos clases de simbolizaciones: 1) la simbolización fonética tradicional y 2) el alfabeto práctico usado por Wright (1974) en su estudio sobre el criollo limonense. Este alfabeto tiene la ventaja de ser fonémico y no requiere el uso de diacríticos. A continuación las unidades del alfabeto práctico con ejemplos ilustradores de las fusiones que han llevado a la simplificación del inventario en mek-a-tél-yu. 


\begin{tabular}{|c|c|c|}
\hline & $|\mathrm{a}|$ & an (and) 'y', bak (back) 'espalda', na (not) 'no' \\
\hline & $|\varepsilon|$ & eg (egg) 'huevo', bredá (brother) 'hermano', we (where) 'dónde' \\
\hline & /I/ & inch (inch) 'pulgada', sik (sick) 'enfermo', wi (we) 'nosotros' \\
\hline 0 & $|a|$ & ógli (ugly) 'feo', bon (burn) 'quemar', nomuo (no more) 'no más' \\
\hline u & $/ U /$ & únu (you) 'ustedes’, buk (book) 'libro', tu (too) 'también’ \\
\hline aa & 101 & aal (all) 'todos', laad (laad (lard) 'manteca', yaa (yes) ‘sí' \\
\hline ii & /i/ & iizi (easy) 'fácil', diip (deep) 'profundo', grii (agree) 'concordar' \\
\hline uu & $\mid u /$ & úufa (whose) 'de quién', fuud (food) 'alimento', bluu (blue) 'azul' \\
\hline ai & /ai/ & ail (oil) 'aceite', nais (nice) 'simpático', bwai (boy) 'muchacho' \\
\hline ie & /ie/ & iet (eight) 'ocho', wiek (wake) 'despertar', pie (pay) 'pagar' \\
\hline ou & la u/ & out (out) 'fuera', kroud (crowd) 'multitud', kou (cow) 'vaca' \\
\hline uo & |ual & uol (old) 'viejo', kuos (course) 'curso', nuo (know) 'conocer' \\
\hline b & /b/ & basilar ( - ) 'gozar', rob (rub) 'frotar', ríba (river) 'río' \\
\hline ch & $|c|$ & choch (church) 'iglesia', rícha (richer) 'más rico' \\
\hline d & $/ d /$ & den (then) 'entonces', wéda (weather) 'tiempo', buod (board) 'tabla' \\
\hline & $|f|$ & fieba /favor) 'favor', shuofá (chofer', nof (enough) 'mucho' \\
\hline g & lg/ & góma (hangover) 'goma', máaga (meager) 'flaco', ag (hog) 'puerco' \\
\hline & /j/ & juok (joke) 'chiste', miejá (major) ‘mayor', boj (budge) ‘mover' \\
\hline k & $|k|$ & kyar (car) 'carro', pakuál (Pacuare) 'Pacuare', luk (look) mirar' \\
\hline I & /1/ & lob (love) 'amar', luonli (lonely) ‘solitario', fuul (fool) ‘tonto’ \\
\hline m & $/ \mathrm{m} /$ & mod (mud) 'lodo', mumá (mother) 'mamá', flim (film) 'película' \\
\hline n & $/ \mathrm{n} /$ & nésio (pest) ‘necio', bránya (bush) 'maleza’, wain (wine) 'vino’ \\
\hline ng & $/ \mathrm{n} /$ & lang (long) 'largo', bánggle (bangle) 'brazalete', onggli (only) 'solamente' \\
\hline $\mathrm{p}$ & $/ \mathrm{p} /$ & patí (meat pie) 'empanada de carne', pupá (father) 'papá, drap (drop) 'caer' \\
\hline r & $|r|$ & rien (rain) 'lluvia', bakrá (white man) ‘hombre blanco', suor (sore) 'llaga’ \\
\hline s & $|s|$ & suo (sew) 'coser', biesn (basin) 'palangana', kies (case) 'estuche' \\
\hline sh & $|s|$ & shúga (sugar) 'azúcar', mashíet 'machete', rosh (rush) 'prisa' \\
\hline $\mathrm{t}$ & $\mid t /$ & tíkya (take care) 'cuidado', moutamási (gossiper) 'chismoso', wie(wait) 'esperar' \\
\hline w & $\mid w /$ & wára (what) 'qué', bwail (boil) 'divieso', pówa (power) 'poder' \\
\hline & $|y|$ & yála (yellow) 'amarillo', gyal (girl) 'muchacha', láaya (lawyer) 'abogado' \\
\hline $\mathbf{z}$ & $|z|$ & zingk (zink) 'cinc', riezá (razor) 'navaja’, gramandáiz (prune) 'podar’ \\
\hline
\end{tabular}

No existen fonológicamente: el fricativo glotal $/ \mathrm{h} /$, el fricativo labidental sonoro $/ \mathrm{v} /$, el fricativo interdental sordo $/ \theta / \mathrm{ni}$ el fricativo sonoro /ð/, $[h]$ y $[v]$, se producen alafónicamente, pero no son rasgos destintivos en el idioma.

Diferencias entre el mek-a-tél-yu y el inglés estándar

Para poder adoptar nuevos hábitos lingü ísticos, el estudiante de un idioma debe saber distinguir los rasgos característicos de las dos lenguas, la propia y la que es objeto de estudio, sean estos fonológicos, morfológicos o sintácticos.

\section{Aspectos fonológicos}

Siguen algunas diferencias fonológicas entre el mek-a-tél-yu y el inglés estándar (S) de origen norteamericano que se enseña en los colegios de Limón:

\begin{tabular}{|c|c|c|c|c|c|c|}
\hline$M$ & $\mathbf{S}$ & & M & $\mathbf{S}$ & & \\
\hline $\begin{array}{l}\text { 1. }|\mathrm{a}| \\
\text { 2. } \mid \mathrm{ie} / \\
\text { 3. }|\mathrm{u} a| \\
4 . \mid \mathrm{ai} / \\
5 . \mid \mathrm{a} / \\
6 . \mid \mathrm{b} / \\
7 . \mid \mathrm{d} / \\
8 . / \mathrm{t} /\end{array}$ & $\begin{array}{l}\text { lael } \\
|\mathrm{e}| \\
10 \mid \\
10 \mathrm{i} / \\
|a| \\
|\mathrm{v}| \\
|\partial| \\
|\theta|\end{array}$ & como en & $\begin{array}{l}\text { man } \\
\text { biebi } \\
\text { uen } \\
\text { ail } \\
\text { ar } \\
\text { fieba } \\
\text { dis } \\
\text { tik }\end{array}$ & 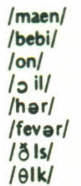 & $\begin{array}{l}\text { (man) } \\
\text { (baby) } \\
\text { (own) } \\
\text { (oil) } \\
\text { (her) } \\
\text { (favor) } \\
\text { (this) } \\
\text { (thick) }\end{array}$ & $\begin{array}{l}\text { 'hombre' } \\
\text { 'bebe' } \\
\text { 'propio' } \\
\text { 'aceite' } \\
\text { 'ella' } \\
\text { 'favor' } \\
\text { 'este' } \\
\text { 'grueso' }\end{array}$ \\
\hline
\end{tabular}

Ejemplos de algunas alternancias:

10. Epéntesis: $\mathrm{S}: \# \mathrm{~s}+$ nasal $=M: \# \mathrm{~s} V+$ nasal

siniek (snake) 'culebra'

sumal (small) 'pequeño'

11. Metátesis: $\mathrm{S}: \mathrm{s}+\mathrm{k} \#=\mathrm{M}: \mathrm{k}+\mathrm{s} \#$

aks (ask) 'preguntar'

12. Apócope de /s/ inicial ante /t/ y /p/: $S$ : $\# s+t(p) V=M: \# t(p) V$ 


\begin{tabular}{|c|c|c|}
\hline $\begin{array}{l}\text { todi } \\
\text { pwail }\end{array}$ & & $\begin{array}{l}\text { (study) } \\
\text { (spoil) }\end{array}$ \\
\hline 13. & $\begin{array}{l}\mathrm{t} \rightarrow \\
\mathrm{d}\end{array}$ & $\varphi / C$ \\
\hline $\begin{array}{l}\text { waan } \\
\text { laas }\end{array}$ & & $\begin{array}{l}\text { (want) } \\
\text { (lost) }\end{array}$ \\
\hline
\end{tabular}

14. Aféresis: Pérdida de /w/ inicial

úman (woman) 'mujer'

15. $|r| \rightarrow \emptyset$ después del núcleo silábico

$\begin{array}{lll}\text { naat } & \text { (north) } & \text { 'norte' } \\ \text { kyaat } & \text { (cart) } & \text { 'carreta' } \\ \text { ton } & \text { (turn) } & \text { 'virar' }\end{array}$

16. Labialización y palatización:

Epéntesis de / $\mathrm{w} /$ entre bilabial y /a/ - Epéntesis de $/ y /$ entre velar $y / a /$ :

\begin{tabular}{|c|c|c|}
\hline $\begin{array}{l}\text { bwai } \\
\text { pwail }\end{array}$ & $\begin{array}{l}\text { (boy) } \\
\text { (spoil) }\end{array}$ & $\begin{array}{l}\text { 'muchacho' } \\
\text { 'estropear' }\end{array}$ \\
\hline $\begin{array}{l}\text { kyat } \\
\text { gyang }\end{array}$ & $\begin{array}{l}\text { (cat) } \\
\text { (gang) }\end{array}$ & $\begin{array}{l}\text { 'gato' } \\
\text { 'pandilla' }\end{array}$ \\
\hline
\end{tabular}

17. Velarización de las oclusivas alveolares ante /I/ silábica final:

$$
\mathrm{t} \rightarrow / \mathrm{k} / \mathrm{o} / \mathrm{g} /
$$

$\begin{array}{lll}\text { bakl } & \text { (bottle) } & \text { 'botella' } \\ \text { migl } & \text { (middle) } & \text { 'medin' }\end{array}$

Un fenómeno afín se nota en onggli (only). La $n$ se velariza ante $/$ y como transición se de la epéntesis de $g$

18. Nasal velar es nasal alveolar en el sufijo -ing: muvin (moving) 'movimiento'

Algunas diferencias sintácticas entre el $\mathrm{M}$ y el $\mathrm{S}$ que se enseña en Limón

1. Las palabras en mek-a-tél-yu no iienen flexiones o conjugaciones finales.

En el ejemplo:

di bwai-dem a-plie (The boys are playing) 'Los muchachos están jugando' la pluralización se lleva a cabo posponiendo el pronombre dem 'ellos'.

2. El presente continuo se marca con a-, y equivale a bet-ing en inglés: a-plie (playing) 'jugando'

3. El verbo no tiene forma pasiva. En lugar de la pasiva del tipo del $\mathrm{S}$, tenemos una constituida por la eliminación obligatoria del agente y la anteposición del objeto al verbo:

di léta rait (The letter was written) 'La carta fue escrita'

4. El adjetivo funciona como núcleo del predicado sin verbo copulativo:

unu liezi (You are lazy) 'Ustedes son perezosos'

5. El auxiliar do no se usa en preguntas:

we dem gaan? (Where did they go?) ¿Adónde fueron?

6. El adverbio de lugar está precedido por el verbo locativo de:

mina de a yaad (Herminia is at home) 'Herminia está en casa' di buk de pan di deks (The book is on the desk) 'El libro está sobre el escritorio'

7. La reduplicación es un proceso productivo en mek-a-tél-yu:

i rien-rien (It rains a lot) 'Llueve constantemente'

im trang-trang (He's very strong) 'El es muy fuerte'

di kyar mashop- mashop (The car is completely wrecked) "El carro está completamente destrozado'

im fain wan-wan grien a koko ( $\mathrm{He}$ found a few grains of cocoa) 'Encontró unos cuantos granos de cacao'

shi biit-biit (She beats constantly) 'Ella castiga constantemente'

im nyam-nyam (He eats continuously) 'El come continuamente'

\section{Consideraciones psicolingüísticas}

¿Cómo se desenvuelve académicamente el hablante de $\mathrm{M}$ en el sistema escolar? iHa sido afectado adversamente en su desarrollo cognoscitivo el hecho que reciba lecciones sólo en español? Herzfeld (1978) hablando de Limón dice: "si tomamos en cuenta las condiciones socioeconómicas adversas que privan en el área, el grado de analfabetismo en Puerto Limón es relativamente bajo comparado con el resto del país". (Extracto traducido) Ver Cuadro 1.

Las investigaciones de Lambert y Tucker (1967) tienden a demostrar que un programa monolingüe en una lengua que no sea la materna no crea 
necesariamente un impedimento, siempre que se maneje como en el experimento que estos dos investigadores llevaron a cabo en las Filipinas. Algunos estudiantes filipinos instruidos totalmente en inglés se desempeñaron académicamente con igual eficiencia que sus compañeros instruidos en tagalo. Los niños instruidos en el idioma inglés lograron asimilar las materias mediante métodos naturales, interactuando diariamente con sus profesores.

El sistema educativo limonense se enfrenta a la ingente tarea de ayudar a sus educandos bilingües a desarrollarse naturalmente, sin inhibiciones y sin menospreciar su tradición lingü ística y cultural. La interferencia de la lengua materna en sus manifestaciones lingüisticas debe ser tratada con mucho tacto, comprensión y tolerancia.

\section{La influencia del inglés norteamericano en Limón}

Dado que la tradición lingüística del negro limonense está ligada a Jamaica, y por ende, a Inglaterra, pareciera lógico que la variedad de inglés que les sería más favorable asimilar es la de un tipo británico. Sin embargo, la influencia del inglés norteamericano ha sido muy fuerte en Limón desde tiempos de la United Fruit Co.: muchos de los jefes eran norteamericanos. También se escuchan con mucha claridad los programas radiales en inglés desde los Estados Unidos (The Voice of America, Armed Forces, Miami, etc.). Durante la temporada de beisbol norteamericano grupos de limonenses toman partido y discuten acaloradamente las "jugadas" de su equipo favorito, oídas por la radio. Además muchos limonenses tienen familiares que emigraron a los Estados Unidos y están en contacto permanente, viajando de allá para acá y vice versa. Debemos tomar en cuenta también que los profesores de inglés son preparados en las universidades del país con profesores y textos de los Estados Unidos. Algunos han estudiado en universidades norteamericanas.

Alguna vez se pensó en preparar libros de texto para la enseñanza del inglés que fueran apropiados para los estudiantes de origen afrocaribeño. Pero este proyecto encontró dura resistencia de parte de padres de familia y de los mismos profesores que se opusieron, alegando entre otras cosas que enseñar un inglés diferente en Limón sería discriminatorio y limitaría las posibilidades de los estudiantes de integrarse a la vida productiva del país. La escasez de profesores idóneos para laborar en el área y la relativamente baja población monolingüe (en $M$ ) fueron otras razones que pesaron en contra del proyecto.

Todo lo dicho pareciera justificar lo que acontece en la actualidad: el uso de textos norteamericanos en la enseñanza en los colegios oficiales de Limón.

\section{El método ecléctico}

El enfoque pedagógico de la enseñanza de idiomas que tiene mayores probabilidades de tener éxito en la enseñanza del inglés a hablantes del $M$ es el ecléctico, vale decir, una teoría basada en una combinación de lo mejor de los diferentes métodos y no basada en uno en particular. A partir de esta teoría se plantean como actitudes necesarias:

1. El énfasis en prácticas de oraciones y frases que tengan sentido.

2. La introducción de la lectura, escritura, y prácticas orales desde el comienzo del curso.

3. La atención a las características del idioma desde el inicio del curso.

4. El énfasis en el significado de las expresiones.

Según Childers (1964) el método opera de la siguiente manera:

1. Se realizan prácticas orales y fonéticas, producción de patrones gramaticales, lectura en voz al ta en las primeras etapas del curso.

2. Se hacen ejercicios de preguntas y respuestas en la lengua que se estudia para evaluar la comprensión oral.

3. Se usan materiales audiovisuales para enseñar el vocabulario y para hablar sobre la cultura y la gente de la lengua en estudio.

4. La gramática se explica deductivamente para ahorrar tiempo.

5. La traducción se usa para determinar si el estudiante comprende lo que lee.

El educador puede no seguir a Childer al pie de la letra, pero podría usar las características que más se adapten a su situación particular. Debe ser lo suficientemente flexible para poder escoger lo mejor de los diferentes métodos.

\section{Conclusión}

El incremento en las investigaciones en la Universidad de Costa Rica y la Universidad Nacional sobre el habla criolla limonense y las lenguas autóctonas indígenas, ha motivado una mejor 
comprensión de los factores sociolingüísticos que operan en situaciones dialectales no estandarizadas. Las autoridades superiores de Educación en nuestro país reconocen la necesidad de implementar nuevos enfoques en estas áreas para que los fines de la educación costarricense puedan ser alcanzados con mayor eficiencia.

Como educadores podemos ayudar al hablante del mek-a-tél-yu a que sea útil a sí mismo y a sus semejantes. Educar es una profesión difícil. Los profesores de las diferentes especialidades tienen dificultades especiales -los de ciencias tienen problemas relacionados con equipos de laboratorio y demostraciones, los de estudios sociales con ilustraciones y documentos, y así sucesivamente.

Los profesores de idiomas tenemos nuestros problemas. Impartimos una materia que es probablemente singular en el sentido de que se puede aprender pero no se puede enseñar. La competencia que los estudiantes alcancen en el uso del idioma la realizan ellos por medio de $s u$ propio esfuerzo, su participación en las prácticas, su comprensión, su inteligencia. Cuanto más aprovechen lo que hacen en el aula, más podrán aprender. Mientras más oportunidades de participar les facilitemos, mejores maestros seremos. Mientras menos hablemos sobre la materia, más tiempo tendremos para los ejercicios en la clase. Nuestras explicaciones casi siempre son o demasiado triviales o muy complicadas. Podemos ayudarlos a asimilar la materia si la presentamos siguiendo una secuencia lógica e interesante y propiciando bastante repaso, sin aburrir a los más listos y $\sin$ desanimar a los más lentos.
Somos modelos y jueces. Modelos para ser imitados y jueces de su actuación. Se requiere paciencia y destreza para corregir las faltas que los alumnos cometerán -inevitablemente- en la lección de inglés. Hay técnicas específicas que pueden ser aplicadas en estos casos. Pero lo más importante no es saber aplicarlas sino saber las razones que las originan.

El producto de un buen curso de idiomas serán unos estudiantes que hayan aprendido a hacer cosas en lugar de saber acerca de cosas.

\section{CUADRO 1}

$\begin{array}{lccc}\begin{array}{l}\text { Provincia } \\ \text { Distrito }\end{array} & \text { Total } & \text { Analfabetos } & \text { Porcentaje } \\ \begin{array}{l}\text { San José } \\ \text { Cantón Central }\end{array} & 509,087 & 33,448 & 6.6 \\ \begin{array}{l}\text { Alajuela } \\ \text { Cantón Central }\end{array} & 70,281 & 6,280 & 8.9 \\ \begin{array}{l}\text { Cartago } \\ \text { Cantón Central }\end{array} & 46,937 & 3,368 & 7.2 \\ \begin{array}{l}\text { Heredia } \\ \text { Cantón Central }\end{array} & 27,854 & 1,034 & 3.7 \\ \begin{array}{l}\text { Guanacaste } \\ \text { Liberia }\end{array} & 15,252 & 1,656 & 10.9 \\ \begin{array}{l}\text { Puntarenas } \\ \text { Cantón Central }\end{array} & 45,262 & 7,071 & 15.6 \\ \begin{array}{l}\text { Limón } \\ \text { Central }\end{array} & 28,895 & 2,272 & 7.9\end{array}$

Fuente: Anita Herzfeld. "Tense and Aspect in Limon Creole: A Sociolinguistic View Towards a Creole Continuum", Diss. The University of Kansas, 1978, p. 63. 


\section{BIBLIOGRAFIA}

Bailey, Beryl L. Jamaican Creole - A Transformational Approach. Cambridge: Cambridge University Press. 1966.

Bailey, Charles-James N. "Some Suggestions for Greater Consensus in Creole Terminology", en Pidgins and Creoles: Current Trends and Prospects. D. DeCamp and I. Hancock, ed. Washington, Georgetown University Press, 1974 , p. 89.

Bickerton, Derek. Dynamics of a Creole Continuum. Cambridge: Cambridge University Press, 1975.

Childers, I. Wesley. Foreign Language Teaching. New York: The Center for Applied Research in Education, Inc. 1964.

De Camp, David. "Analysis of a Post-Creole Contiuum", en Pidginization and Creolization of Languages. Dell Hymes, ed. New York: Cambridge University Press, 1971, pp. 349-370.
Herzfeld, Anita. "Tense and Aspect in Limon Creole: A Sociolinguistic View Towards a Creole Continuum". The University of Kansas. Disertación sin publicar, 1978.

Twaddell, W. Freeman. "Recent Trends in Foreign Language Teaching", en English Education in Japan. ELEC Publications Vol. X (Tokyo: ELEC, 1977), pp. 15-41.

Wilson, Jack L. "Análisis fonológico del bribri”, en América Indígena. México. Instituto Indigenista Interamericana, 1973.

Wright, Fernando. "Limon Creole: A Syntactic Analysis". San José, Costa Rica: Universidad de Costa Rica. Tesis de Licenciatura sin publicar. 1974.

"Problems and Methods of Teaching English as a Second Language to Limon Creole Speakers". The University of Kansas. Tesis de Maestría sin publicar, 1979. 
